\title{
Quantitative assessment of intragenic receptor tyrosine kinase deletions in primary glioblastomas: their prevalence and molecular correlates
}

\author{
Edward R. Kastenhuber $\cdot$ Jason T. Huse $\cdot$ Samuel H. Berman • Alicia Pedraza \\ Jianan Zhang $\cdot$ Yoshiyuki Suehara $\cdot$ Agnes Viale $\cdot$ Magali Cavatore $\cdot$ Adriana Heguy $•$ \\ Nicholas Szerlip · Marc Ladanyi · Cameron W. Brennan
}

Received: 25 February 2013 / Revised: 10 November 2013 / Accepted: 13 November 2013 / Published online: 29 November 2013 (C) The Author(s) 2013. This article is published with open access at Springerlink.com

\begin{abstract}
Intragenic deletion is the most common form of activating mutation among receptor tyrosine kinases (RTK) in glioblastoma. However, these events are not detected by conventional DNA sequencing methods commonly utilized for tumor genotyping. To comprehensively assess the frequency, distribution, and expression levels of common RTK deletion mutants in glioblastoma, we analyzed RNA from a set of 192 glioblastoma samples from The Cancer Genome Atlas for the expression of EGFRvIII, EGFRvII, EGFRvV (carboxyl-terminal deletion), and PDGFRA $\triangle 8,9$. These mutations were detected in $24,1.6,4.7$, and $1.6 \%$ of cases, respectively. Overall, $29 \%(55 / 189)$ of glioblastomas expressed at least one RTK intragenic deletion transcript in this panel. For EGFRvIII, samples were analyzed by both
\end{abstract}

E. R. Kastenhuber and J. T. Huse have contributed equally to this work.

Electronic supplementary material The online version of this article (doi:10.1007/s00401-013-1217-3) contains supplementary material, which is available to authorized users.

E. R. Kastenhuber $\cdot$ J. T. Huse $\cdot$ Y. Suehara $\cdot$ M. Ladanyi Department of Pathology, Memorial Sloan-Kettering Cancer Center, New York, NY, USA

J. T. Huse $\cdot$ J. Zhang $\cdot$ M. Ladanyi $\cdot$ C. W. Brennan Brain Tumor Center, Memorial Sloan-Kettering Cancer Center, New York, NY, USA

S. H. Berman · A. Pedraza · Y. Suehara - A. Heguy · M. Ladanyi ·

C. W. Brennan $(\square)$

Human Oncology and Pathogenesis Program,

Memorial Sloan-Kettering Cancer Center, New York, NY, USA

e-mail: cbrennan@mskcc.org

A. Viale $\cdot$ M. Cavatore

Genomics Core Facility, Memorial Sloan-Kettering Cancer

Center, New York, NY, USA quantitative real-time PCR (QRT-PCR) and single mRNA molecule counting on the Nanostring nCounter platform. Nanostring proved to be highly sensitive, specific, and linear, with sensitivity comparable or exceeding that of RNA seq. We evaluated the prognostic significance and molecular correlates of RTK rearrangements. EGFRvIII was only detectable in tumors with focal amplification of the gene. Moreover, we found that EGFRvIII expression was not prognostic of poor outcome and that neither recurrent copy number alterations nor global changes in gene expression differentiate EGFRvIII-positive tumors from tumors with amplification of wild-type EGFR. The wide range of expression of mutant alleles and co-expression of multiple EGFR variants suggests that quantitative RNA-based clinical assays will be important for assessing the relative expression of intragenic deletions as therapeutic targets and/or candidate biomarkers. To this end, we demonstrate the performance of the Nanostring assay in RNA derived

\footnotetext{
A. Heguy

Geoffrey Beene Translational Oncology Core Facility,

Memorial Sloan-Kettering Cancer Center, New York, NY, USA

N. Szerlip

Department of Neurosurgery, Wayne State University Medical

School, Detroit, MI, USA

C. W. Brennan

Department of Neurosurgery, Memorial Sloan-Kettering Cancer

Center, New York, NY, USA
} 
from routinely collected formalin-fixed paraffin-embedded tissue.

Keywords EGFRvIII - GBM · Glioblastoma · Nanostring $\cdot$ RNA sequencing $\cdot$ TCGA

\section{Introduction}

Large-scale genomic characterization has confirmed striking heterogeneity underlying the molecular landscape of GBM and has catalogued a spectrum of tumor suppressors and oncogenes affected by deletion, amplification, mutation, and/or rearrangement. Alterations of receptor tyrosine kinases (RTKs) are especially prevalent in GBM. RTKs are a class of mitogenic signaling proteins including epidermal growth factor receptor (EGFR), platelet-derived growth factor receptor- $\alpha$ (PDGFRA) and MET, that are widely implicated in human oncogenesis. Indeed, highlevel amplification of the EGFR locus represents the single most common genomic abnormality in GBM, occurring in $\sim 45 \%$ of all cases, and PDGFRA and MET are also frequently amplified, in $10-15 \%$ and $\sim 4 \%$ of GBMs, respectively [5, 10, 31, 43]. Moreover, these amplification events have been associated with specific disease subclasses, defined by transcriptional and proteomic signatures [4, 37, 45], implying that molecular distinctions within GBM are, to some extent, mechanistically grounded in dysregulated RTK signaling.

RTK amplification in GBM is often associated with intragenic deletions and gene rearrangements, as well as extracellular domain point mutations [5, 23, 44]. As many as half of EGFR-amplified GBMs have been reported to express the variant III mutation (vIII), a 287-amino acid in-frame deletion of exons 2-7 in the EGFR extracellular domain (ECD) [42]. The resulting protein constitutively signals in a ligand-independent manner by forming homodimers or heterodimeric complexes with either wildtype EGFR or other ErbB family members [12]. EGFRvIII primarily stimulates the oncogenic PI3K/AKT pathway $[17,29]$, but is also known to interact with the adapter proteins Shc and Grb2, thereby activating RAS/MAPK signaling [39]. Additionally, EGFRvIII-expressing tumor cells may exert paracrine influence on their neighbors by secreting either microvesicles containing the protein itself [1] or mitogenic cytokines like IL-6 and LIF [19]. Other cancerrelevant functionalities ascribed to EGFRvIII include evasion of apoptosis [30], tumor cell invasion [22], angiogenesis [50] and stem cell self-renewal [16].

A number of additional EGFR intragenic deletions have been identified. Some, like EGFR vI (exon 1-7 deletion) and EGFR vIV (intracellular domain microdeletion), are rare $[6,9,38,48]$, while others like EGFRvII and EGFRvV are marginally more common, each accounting for more than $10 \%$ of all GBM-associated EGFR mutations [20, 28, 32]. The vII deletion includes a small 83-amino acid stretch within the EGFR ECD [47], while EGFRvV involves a C-terminal truncation that ablates the majority of the protein's intracellular domain, a region responsible for mediating internalization and degradation [6, 9, 48]. Functional analyses of both mutations have been complicated by their frequent co-occurrence with EGFRvIII [10]. However, recent work has demonstrated that EGFRvV is itself capable of transformation both in vitro and in mouse xenografts [7].

Intragenic rearrangements in PDGFRA have also been described in GBM. Similar to their counterparts in EGFR, these appear to largely occur in the context of high-level genomic amplification. An in-frame deletion in the Ig-like, extracellular domain of PDGFRA (PDGFRA $\Delta 8,9$ ) has been detected in up to $40 \%$ of PDGFRA-amplified cases and results in constitutive kinase activation in vitro [21,36]. Cases of C-terminal truncation (PDGFRA $\Delta \mathrm{Ct}$ ) have also been reported, although defined functional consequences remain to be established [40]. Moreover, it has yet to be determined how these mutations correlate with other oncogenic and subclass-defining molecular abnormalities in GBM.

The prevalence of RTK intragenic deletions, particularly EGFRvIII, in significant subsets of GBM has made them both attractive therapeutic targets for immunotherapeutic approaches and promising predictive biomarkers for pharmacologic receptor inhibitors [26, 35]. In this context, there remains a need to effectively detect and quantify EGFR vIII and related abnormalities in RTKs to power more detailed functional analysis and therapeutic trial stratification. Currently, most clinical labs that assess EGFRvIII status do so using non-quantitative techniques such as immunohistochemistry (IHC) and/or reverse transcription-polymerase chain reaction (RT-PCR) for the mutant transcript. Other intragenic deletions in EGFR and those of PDGFRA are not routinely measured as a component of standard patient care.

To determine the frequency and molecular context of common RTK intragenic deletions in GBM, we profiled 192 tumors from TCGA for EGFRvIII using both quantitative reverse transcriptase PCR (QRT-PCR) and a novel approach based on Nanostring nCounter technology. The latter platform was also employed to assess EGFRvII, EGFRvV, and PDGFRA $\Delta 8,9$, in the same sample set. We demonstrate that intragenic deletion mutants, particularly EGFRvIII, comprise highly variable proportions of total RTK expression in a given tumor, ranging from the majority mRNA species to only a minor component. Paired with orthogonal profiling data from TCGA, these findings now represent the most comprehensive tumor-based assessment of RTK deletion mutation in GBM to date, and provide a resource for integrated molecular analysis. Moreover, we find that Nanostring-based analysis performs robustly 
from formalin-fixed paraffin-embedded tissue (FFPE), thus empowering investigation and characterization of a wide dynamic range of expression of EGFRvIII and other deletion mutations in the context of clinical trials.

\section{Methods}

\section{Human tissue and RNA extraction}

RNA from TCGA samples was allocated from the Biospecimen Core Resource as $3 \mu \mathrm{g}$ aliquots and sent to the MSKCC TCGA Pilot Phase Cancer Genome Characterization Center (CGCC). TCGA sample collection and RNA extraction followed published protocols [5, 44]. An additional independent tumor sample set was used to confirm the fidelity of the assay applied to FFPE, including surgical specimens collected at Memorial Sloan-Kettering Cancer Center and frozen. All patients consented prior to surgery under a protocol approved by the institution's Institutional Review Board. Patient-matched FFPE tissue for comparison was obtained following routine processing by the Department of Pathology and diagnostic confirmation by a neuropathologist (J.T.H.). RNA was extracted from either crushed frozen tissue or 3-8 $10 \mu \mathrm{m}$ slides using the RNeasy Mini kit (Qiagen).

\section{Quantitative reverse transcriptase PCR}

From the TCGA sample set, 275 cases with available RNA were interrogated for relative expression of wild-type EGFR and EGFRvIII by RT-PCR. 400 ng of total RNA was reverse-transcribed using the Thermoscript RT-PCR system (Invitrogen) at $52{ }^{\circ} \mathrm{C}$ for $1 \mathrm{~h} .20 \mathrm{ng}$ of resultant cDNA was used in a Q-PCR reaction using an 7500 Real-Time PCR System (Applied Biosystems) and custom-designed TaqMan gene expression Assays (EGFRvIII Forward primer: 5'CGGGCTCTGGAGGAAAAG3'; EGFRvIII reverse primer: 5'AGGCCCTTCGCACTTCTTAC3'; EGFRvIII internal primer: $5^{\prime}$ GTGACAGATCACGGCTCGTG3 $^{\prime}$; total EGFR: pre-designed TaqMan ABI Gene expression Assays Hs01076076_m1). Primers were chosen based on their ability to span the most $3^{\prime}$ exon-exon junction. Amplification was carried for 40 cycles $\left(95{ }^{\circ} \mathrm{C}\right.$ for $15 \mathrm{~s}, 60{ }^{\circ} \mathrm{C}$ for $1 \mathrm{~min}$ ). To calculate the efficiency of the PCR reaction, and to assess the sensitivity of each assay, we also performed a six-point standard curve $(5,1.7,0.56,0.19,0.062$, and $0.021 \mathrm{ng}$ ). Triplicates CT values were averaged, amounts of target were interpolated from the standard curves and normalized to TBP (TATA box binding protein pre-designed TaqMan ABI Gene expression Assays Hs00427620_m1). Efficiency of each reaction was determined from the standard curve of a serially diluted sample using the equation:
Efficiency $=10^{(-1 / \text { slope })}-1$, where slope is fitted to CT vs. $\log 10$ (concentration). Relative quantities of TBP, EGFR and EGFRvIII were calculated from each CT[i] based on the reaction efficiencies and minimum CTs from the standard dilution curves $\left(\mathrm{CT}_{\max }\right)$ according to the formula: Quantity $=(1+\text { Efficiency })^{(\mathrm{CTmax}-\mathrm{CT})}$. All reactions were performed in triplicate. Samples were rejected if multiple TBP replicates failed to cross threshold in $<36$ cycles or if the median absolute deviation of quantified TBP across replicates was greater than $25 \%$ ( 5 of 275 samples). The relative quantities of EGFR and EGFRvIII were normalized with respect to TBP.

\section{Nanostring}

The nCounter Analysis System (Nanostring Technologies, Seattle, WA) allows for multiplexed digital mRNA profiling without amplification or generation of cDNA [13]. Briefly, mRNA is hybridized with pairs of $\sim 50$ bp probes complementary to each target. The reporter probe is tagged by a target-specific code of four fluorescent reporters at seven positions along a phage DNA backbone. The capture probe is used for immobilization on a slide and once oriented in an electric field; bound reporters are counted and annotated. A custom probe set was designed as detailed in Supplemental Table S1. Total RNA (150-300 ng) was hybridized with the codeset probes and loaded into the nCounter prep station. The samples were quantified using the nCounter Digital Analyzer.

The Nanostring platform includes negative control probes (not complementary to any endogenous mRNA) to assess background noise associated with the fluorescent barcode optical recognition system. To ensure that all samples were within the optimal range of probe density for image analysis, we confirmed that there was no systemic increase in negative control counts as a function of total number of counts recorded per sample. Raw probe counts were normalized to a panel of 8 control genes (B2M, B4GALT1, CLTC, E2F4, GAPDH, POLR2A, SDHA, and TBP) by taking the ratios of each gene's counts per sample to the average across all samples and scaling by the median of these ratios in each sample. This normalization factor was also applied to the negative control probes counts. A detection threshold was defined for each sample as five times the mean of the negative control probe normalized counts. Of 192 samples run, three cases (TCGA-02-0021, TCGA-12-0827 and TCGA-19-1386) were excluded from analysis as outliers with low expression of the 8 control genes (possibly representing under-loading or poor hybridization).

C-terminal deletion mutation was inferred by the occurrence of relative underexpression (undercounting) of the exon 28 probe versus the exon 19 probe. The normal 
(wild-type) linear relationship of counts between these two probes was determined by a linear model fit to the central $90 \%$ of the data. This model was then applied to the entire dataset to identify cases with outlier C-terminal underexpression. These cases fell into in two groups: intermediate expression of the truncation mutant $(<60 \%$ of expected c-terminal counts), or high expression $(<10 \%)$.

RNA and DNA sequence analysis

RNA and DNA sequencing data (BAM files mapped to hg19) were obtained from TCGA through CGHub. RNA sequencing was analyzed to tabulate EGFR and PDGFRA exon junctions as described [5]. Briefly, counts were made of all EGFR and PDGFRA reads spanning exon-exon junctions and all paired exonic reads with gaps spanning one or more introns. Only reads with perfect alignment scores (CIGAR score) were considered. To account for $3^{\prime}$ bias in RNA sequence representation, mutant junction counts were compared with counts of normal junctions at the $3^{\prime}$ exon. For example, EGFRvIII expression was defined by counting reads with E1-E8 junctions and comparing to the count of reads with "wild type" E7-E8 junctions. EGFRvII was defined by E13-E16 vs. wild-type E15-E16. PDGFRA D89 was defined by E7-E10 vs. wild-type E9-E10. A junction was counted only if seen in more than one read. Exome DNA sequence data for 291 tumors were analyzed to determine read coverage within the EGFR gene in two regions: exons 2-7 (the EGFRvIII deleted region) and exons 8-22 (spanning the transmembrane and kinase domain regions). The normal ratio of counts between regions was determined by linear regression fit of the middle $90 \%$ of ratios. This model was applied to normalize the ratios and allow accurate estimation of relative copy number of exons $2-7$ vs. exons 8-22.

\section{DNA copy number analysis}

TCGA Level 3 copy number data (normalized and segmented) were downloaded from the TGCA Data Portal for Affymetrix SNP6.0 data (Broad Institute). Copy number was inferred for exon 6 (within the 2-7 deletion) and compared with that of exon 19 (kinase domain region) to identify relative deletion. Level 2 data (normalized) for Agilent 244k aCGH data (MSKCC) were downloaded parsed into to subsets of probe values: probes residing between the midpoint of intron 1 and the endpoint of exon 7 were taken as representing the deleted region in VIII and these $\log 2$ ratios were compared to those of probes residing from the start of exon 8 through exon 21 using Student's $t$ test. A $p$ value of 0.05 was taken as significant (uncorrected for multiple testing). CNA focality, a measure of how many genes are included in simple and complex aberrations, was scored for EGFR in each sample using a Genome Topography Scan method previously described (GTS [5, 43, 49]).

Statistical analysis

Data analyses were performed in $\mathrm{R}$ (http://cran. r-project.org/). A prospective panel of hypotheses regarding the difference between EGFR-amplified/EGFRvIII+ and EGFR-amplified/EGFRvIII- were evaluated by Fisher's exact test for discrete events and by a two-sided student's $t$ test for continuous variables and $p$ values were adjusted by FDR. In all cases, the EGFRvIII-high and low positives (EGFRvIII in $>1 \%$ of EGFR transcripts), along with EGFR-high positives alone (EGFRvIII in $>10 \%$ of EGFR transcripts), were independently compared with wild-type EGFR-amplified tumors. Exploratory searches for differentially expressed genes and miRNAs were performed using empirical Bayes analysis within the Linear Models for Microarray Analysis package implemented in R [41].

\section{Results}

QRT-PCR and Nanostring profiling reveal a wide range of EGFRvIII expression in EGFR-amplified GBM

To assess the frequency and extent of EGFRvIII mutations in GBM, we developed two independent methods for quantitative measurement of EGFRvIII. After initial validation, these assays were applied to mRNA extracted from GBM samples as part of the initial GBM TCGA Pilot Project [5, 44]. Specifically, a TaqMan-based qRT-PCR approach was compared to a Nanostring nCounter assay (NS), each targeting both the exon 1-8 junctional region of EGFRvIII (E1-8) and the EGFR kinase domain (KD) as well as select control genes (see "Methods"). After normalization, EGFRvIII expression (E1-8) was compared to total EGFRencoding mRNA (KD). The expression of EGFRvIII was categorized using NS as absent (<fivefold above mean negative control counts, see "Methods") or present as a fraction of overall EGFR: $<1 \%$ (black), 1-10\% (orange) or $\geq 10 \%$ (red). As shown in Fig. 1a, b, both platforms demonstrated a similar pattern of expression of EGFR overall (KD) and of the vIII variant ranging over three orders of magnitude. EGFRvIII measures were well correlated across platforms (Fig. 1c). Notably, this correlation was seen even among cases with NS counts below the negative detection threshold (open circles, Fig. 1c) suggesting either detection of very low levels of EGFRvIII expression or a component of non-specific hybridization common to both platforms. The estimated ratio of EGFRvIII to total EGFR was highly concordant between platforms (Fig. 1d). Linearity of the Nanostring readout was confirmed by performing 

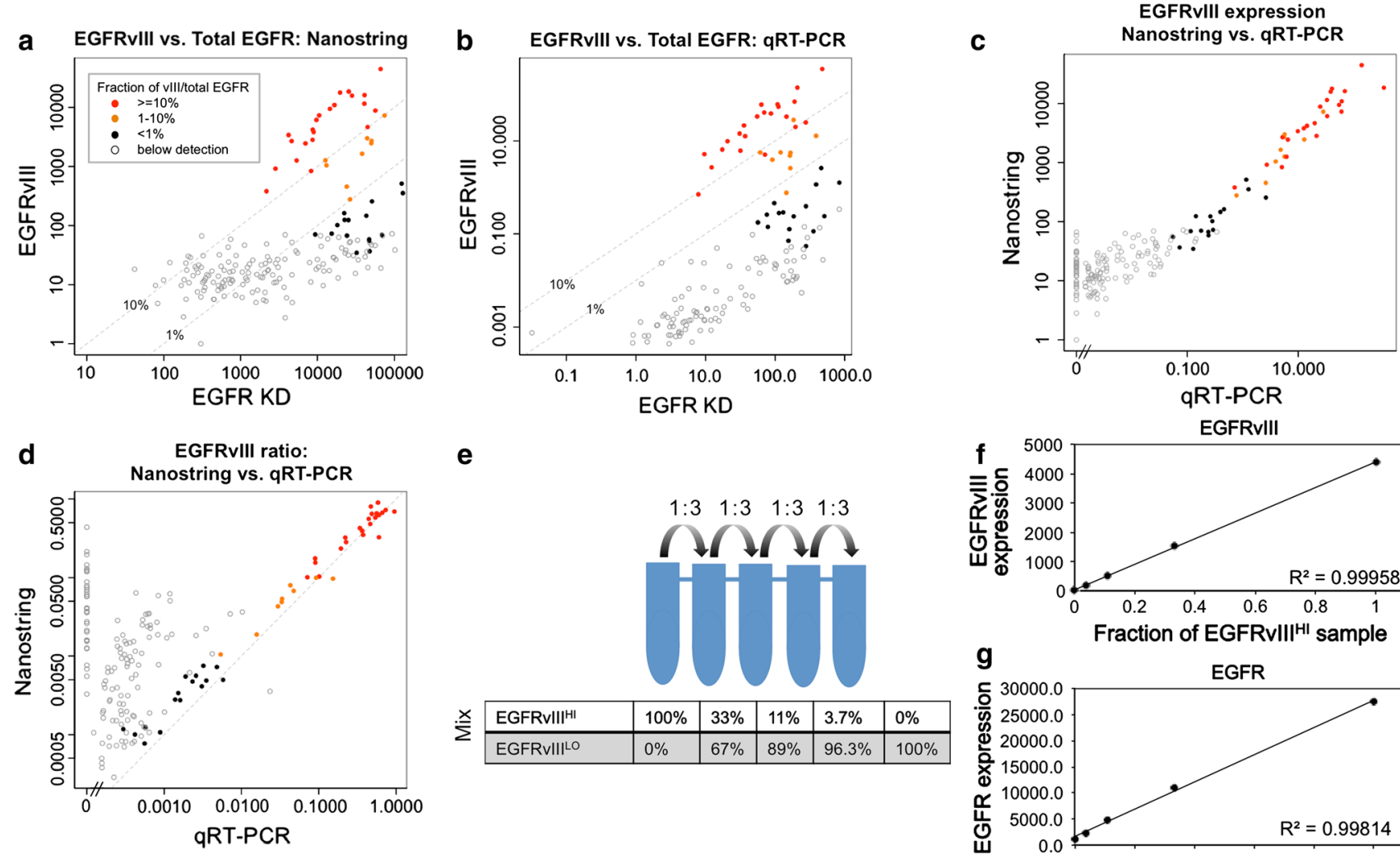

e
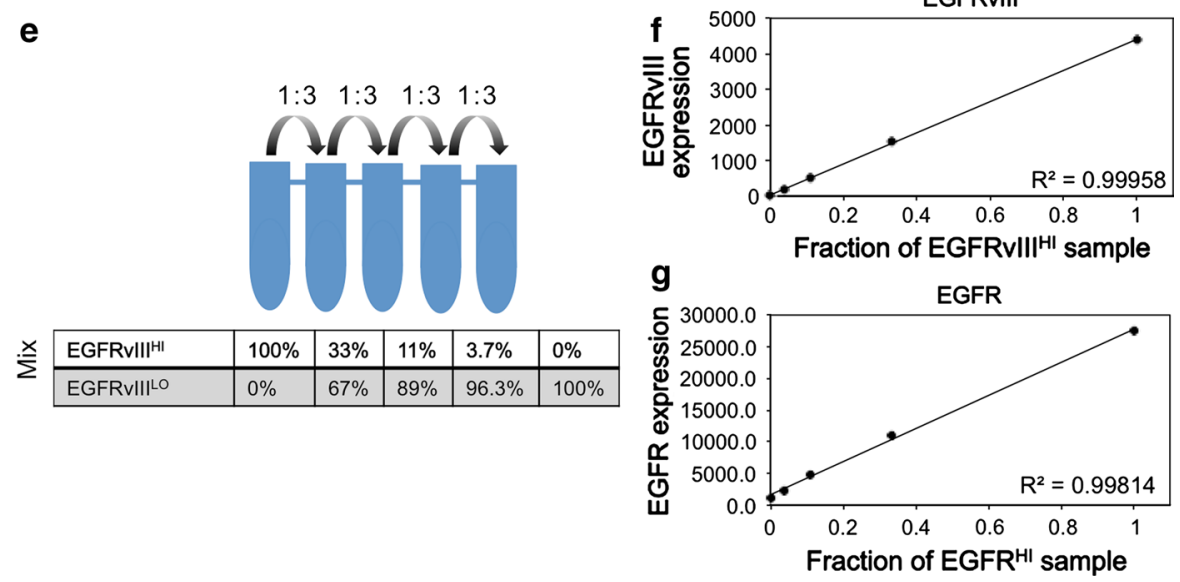

Fig. 1 Expression of EGFRvIII as a fraction of total EGFR is quantified by Nanostring assay and qRT-PCR in 189 GBMs. a Expression of EGFRvIII (exon 1-8 junctional probe) is shown as a function of EGFR kinase domain $(K D)$, determined by normalized Nanostring $(N S)$ counts. Expression levels are classified as high [red mutation in $>10 \%$ transcribed allelic fraction $(T A F)$ ], intermediate (orange 1-10 \% TAF), marginal (black $<1 \%$ TAF) or negative (open circles). These color assignments are carried through panels b-d. b Correlation of EGFRvIII expression between NS and qRT-PCR. Normalized expression levels are plotted for EGFRvIII and KD from the

serial dilution of an EGFR vIII-high positive sample into an EGFR vIII-negative sample, yielding near-perfect correlation $\left(R^{2}>0.99\right)$ (Fig. 1e-g).

Validation of the Nanostring assay with RNA-seq

To more definitively ascertain the performance of the Nanostring assay, we correlated our findings with transcriptome sequencing (RNA-seq) data from TCGA, available for 61 samples in our study set ( 47 with NS data). We found near universal agreement between "positive" status on the Nanostring platform and the presence of bridging RNAseq reads spanning the exon 1-8 breakpoint in EGFRvIII (Fig. 2a, colored dots). Only one discordant sample, judged positive by Nanostring but lacking confirmed junctional reads by RNA-seq, was identified (Fig. 2a, arrow). However, this absence of reads is within sampling error based
Taqman assay (see "Methods"). Samples are colored according to NS expression classification from Fig. 1a. c Cross-platform correlation of EGFRvIII epression, NS vs. qRT-PCR. d Cross-platform correlation of EGFRvIII as a fraction of total EGFR, NS vs. qRT-PCR. e Experimental design of dilution experiment to establish linearity of the Nanostring assay. A sample with high relative expression of EGFRvIII was diluted with a sample negative for EGFRvIII expression, maintaining a constant $250 \mathrm{ng}$ of total RNA in each reaction. $\mathbf{f}$ Counts of EGFRvIII and EGFR KD as a function of diluted fraction of EGFRvIII-containing sample

on the RNA-seq coverage $(225 \times)$ given transcribed allelic fraction of VIII at $1.6 \%$ as estimated by NS. RT-PCR confirmed that this discrepant case was EGFRvIII+, with a low transcribed allelic fraction ( 9\%). Overall, the coverage of counts by NS was much higher than reads by RNA-seq. Among the 47 samples with both RNA sequencing and NS data, the mean coverage of EGFR by RNA-seq was $450 \times$ compared to a mean of 12,000 counts in the NS data. Overall, $60 \%$ of samples, comprised largely of tumors without high-level EGFR amplification, did not reach $100 \times$ coverage at the EGFR locus by RNA-seq, precluding the definitive detection of a minor $(<1 \%)$ transcript population.

To estimate a lower bound for EGFRvIII expression among samples with relatively low EGFR expression, we pooled all RNA-seq reads from 25 cases with NS counts for EGFR <10,000 and EGFRvIII counts up to 100 (box, Fig. 2a). Among a total of 853 reads of EGFR, the vIII 


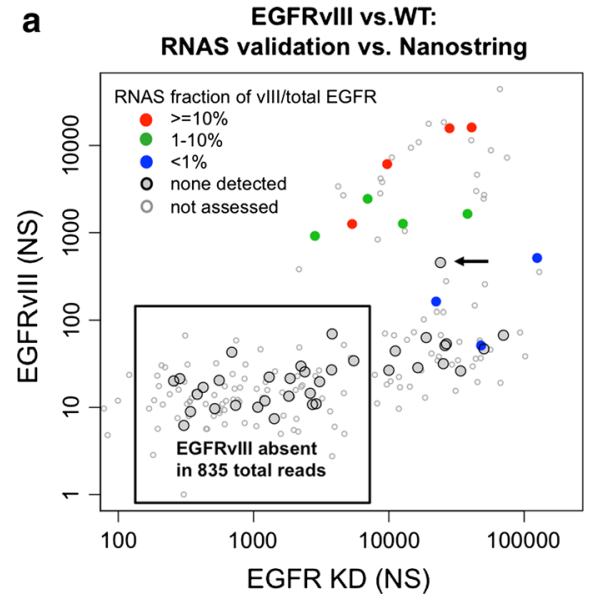

b

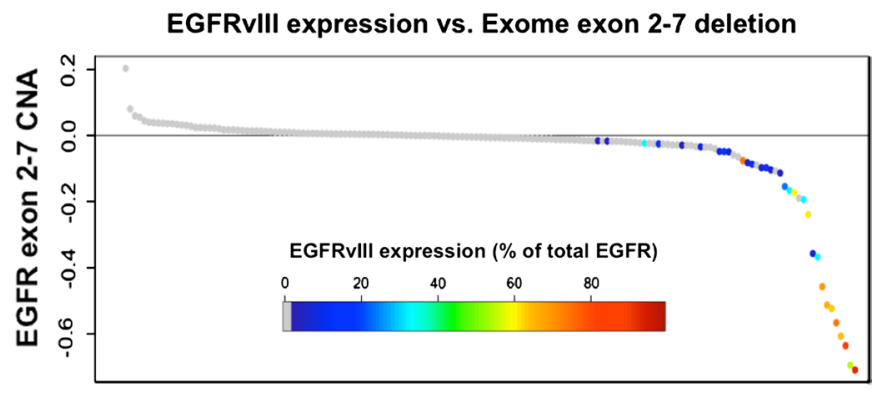

Fig. 2 Comparison with orthogonal platforms a EGFRvIII vs. total EGFR as determined by Nanostring is plotted. EGFRvIII expression was determined independently from TCGA RNA-seq analysis (RNAS). Red denotes cases with $>10 \%$ TAF by RNAS, green $1-10 \%$ and blue $<1 \%$. Black circles filled with gray mark cases where no RNAS reads identified EGFRvIII; empty circles mark cases

junction was not seen once, suggesting that any expression of EGFRvIII in this population is $<1 \%$ at most (95\% confidence interval is $0-0.43 \%$ ).

Because EGFRvIII mutation is associated with genomic deletion of exons 2-7, we sought to determine whether assessment of this deletion by DNA copy number data could serve as a surrogate for RNA-based determination. TCGA exome sequencing data for $291 \mathrm{GBMs}$ were analyzed to determine the read coverage within the EGFR exon 2-7 interval and a control interval from exon 8-22 (see "Methods"). EGFRvIII expression was then compared for 157 cases for which RNA-seq data were also available. As shown in Fig. 2b, all samples that expressed EGFRvIII mRNA showed evidence of a relative loss of exons 2-7 at the DNA level, and samples with the greatest copy number change showed the highest vIII expression levels. Exon 2-7 deletion inferred from exome coverage was able to predict vIII expression with at least $80 \%$ sensitivity at $95 \%$ specificity (Supplemental Fig. S1a). In the TCGA dataset, exome coverage was a more sensitive detector of intragenic deletion than microarray data, specifically the Affymetrix SNP6.0 and Agilent array-CGH platforms, although all DNA measures lacked the sensitivity of mRNA assays (Supplemental Fig. S1b, c).

Nanostring profiling effectively detects EGFRvII, EGFRvV, and PDGFRA $\Delta 8,9$ in small subsets of RTK-amplified GBM

Using analogous approaches to that employed for EGFR vIII, we developed Nanostring assays for the detection of EGFR vII and PDGFRA $\Delta 8,9$ based on their for which RNA-seq data were unavailable. b EGFRvIII expression was compared with genomic loss of EGFR exons 2-7 in 157 cases for which both RNA and DNA (exome) sequencing data were available. Samples are ordered by the magnitude of exon 2-7 deletion inferred from DNA seq coverage. Expression was determined by the ratio of VIII junction RPKM to total EGFR

specific breakpoint regions. Additionally, we sought to measure EGFR $\mathrm{vV}$ transcript by including a probe set in our Nanostring panel directed against the C-terminal of EGFR (EGFR C-term), allowing detection based on the count ratio of the $\mathrm{C}$-term and kinase domains.

Applying these assays to the TCGA cohort revealed distinct clusters of outliers characterized by high-level expression of mutant transcript (Fig. 3a-c). For EGFRvII, we detected three samples expressing the mutant allele over a threshold of $2 \%$ of total EGFR counts (and with EGFRvII count $>5 \times$ negative controls). RNA-seq data were available for two of the three cases and confirmed expression of the vII junction in both (Supplemental Fig. S2). Although NS data demonstrated a strong correlation between total EGFR expression and a low level $(<1 \%)$ of EGFRvII counts, RNA-seq failed to confirm the vII junction in most of these cases (Supplemental Fig. S2).

For EGFR vV, we stratified positive samples into "high" and "low" on the basis of percent composition of C-terminal deleted transcript (Fig. 3b, see "Methods"). Five cases, accounting for $2.6 \%$ of all tumors and $6 \%$ of the EGFRamplified subset, exhibited marked C-terminal loss ( $>90 \%$ EGFR mutated; Fig. 3b, red). Interestingly, a recent TCGA report examining genomic alterations in EGFR by microarray-based copy number analysis demonstrated that these same five samples exhibit profoundly reduced levels of the EGFR C-terminal exon [7]. Moreover, our data also indicated lower expression levels of the C-terminal deletion transcript in four previously unidentified samples (Fig. 3b, orange). Taken together, $4.7 \%$ of cases overall $(10.8 \%$ of EGFRamplified cases) showed evidence of $\mathrm{C}$-terminal truncation 

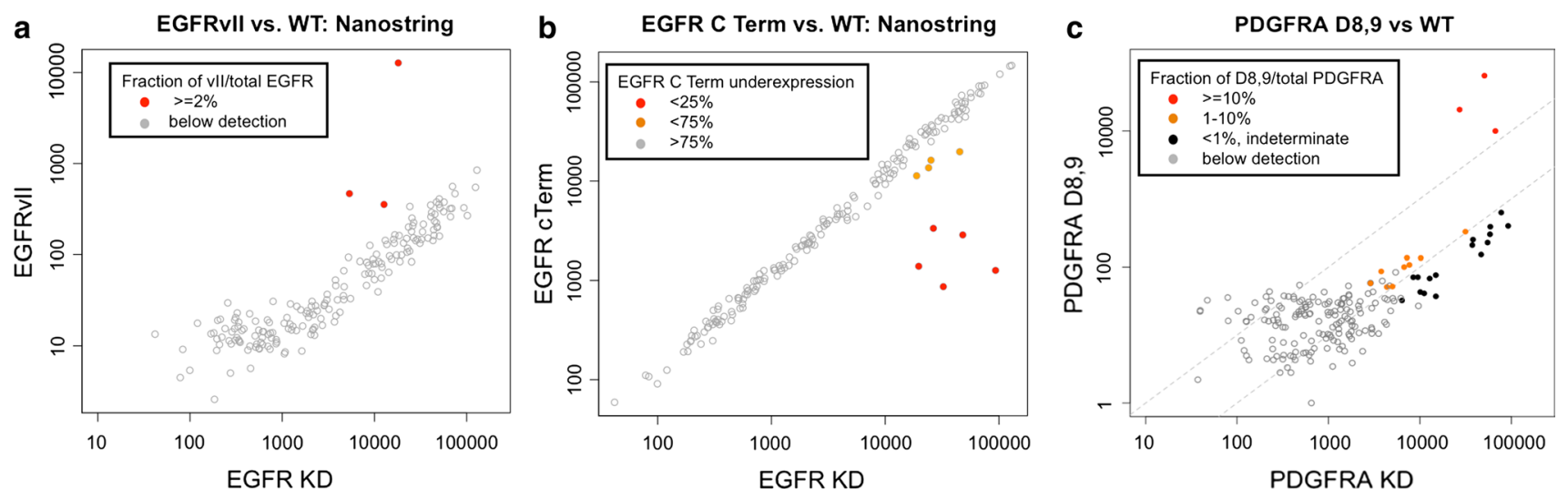

Fig. 3 Assessment of EGFRvII, EGFRvV and PDGFRA $\Delta 8,9$ using Nanostring probes. a Probes targeting the aberrant junctions characterizing EGFRvII expression levels are classified as positive (red mutation in $>2 \% \mathrm{TAF}$ ), or not detected (open circles). b EGFRvV
(C-terminal deletion) is detected by relative under-representation of exon 28 vs. exon 19 harboring the kinase domain $(K D)$. c PDGFRA $\Delta 8,9$ expression is stratified as in Fig. 1a

correlations for the population as a whole. The NS assay also appeared to perform well in the context of presumptive clinical decision-making. Specifically, a binary classifier for EGFRvIII (negative/borderline versus positive) applied to results from FFPE material demonstrated $100 \%$ sensitivity and $94 \%$ specificity (Fig. 4c). Moreover, measured EGFRvIII counts in the two identified "false positives" were in the low-positive range, indicating superior performance in samples containing high levels of EGFRvIII transcript. As confirmation of the specificity of the Nanostring assay for EGFRvIII in gliomas, no positive results were observed in RNAs from 269 non-glioma samples (not from TCGA) including 97 lung adenocarcinomas, 23 ductal breast carcinomas, 36 colon carcinomas, 21 thyroid carcinomas, 25 osteosarcomas, 12 chondrosarcomas, 18 cholangiocarcinomas, and 37 samples of non-neoplastic lung tissue (Y. Suehara, M. Ladanyi, unpublished data). Concordance between FFPE and frozen was comparable for the other deletion mutation probes (Supplemental Fig. S3).

Consistent with extensive prior literature [12, 42, 51], we found a tight association between EGFRvIII mutation and high-level EGFR amplification in our sample set defined by aCGH $\log 2$ ratio $>2$ (Fig. 5a). Only two cases with highlevel EGFRvIII expression demonstrated $\log 2$ ratios below 2 (TCGA-06-0156 and TCGA-08-0360). However, examination of aCGH data for both cases revealed focal CNA of the EGFR locus in a pattern consistent with high-level amplification within a subpopulation of cells, as confirmed by FISH for one sample (TCGA-06-0156) [43]. We confirmed this observation by evaluating all 116 cases with both RNA-seq and aCGH data available (Supplemental Fig. S4). EGFRvIII transcript was detected by sequencing in 38 of the 64 cases with focal EGFR amplification (59\%). No EGFRvIII transcript was found among the subset of 52 unamplified cases, while the WT junction was read a total sequently, the variance of noise at near-zero counts reduced 

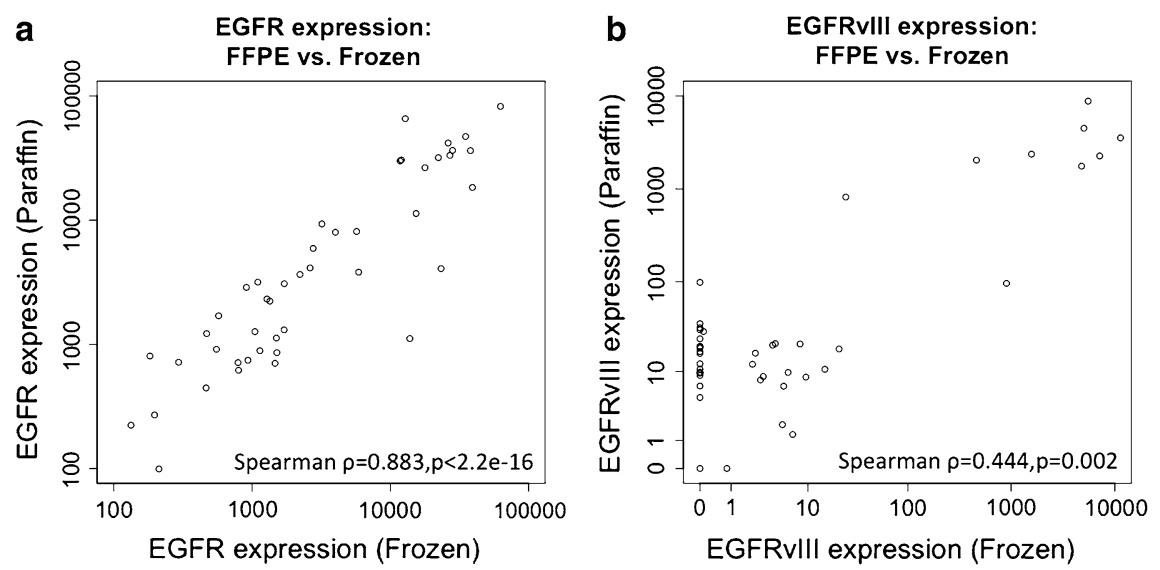

C

\begin{tabular}{|c|c|c|}
\hline & $\begin{array}{c}\text { EGFRvIII- } \\
\text { (Frozen) }\end{array}$ & $\begin{array}{c}\text { EGFRvIII+ } \\
\text { (Frozen) }\end{array}$ \\
\hline $\begin{array}{c}\text { EGFRvIII- } \\
\text { (FFPE) }\end{array}$ & 34 & 0 \\
\hline $\begin{array}{c}\text { EGFRvIII+ } \\
\text { (FFPE) }\end{array}$ & 2 & 9 \\
\hline
\end{tabular}

Fig. 4 Performance of Nanostring assay applied to suboptimal material. Counts of EGFR-WT (a) and EGFRvIII (b) are correlated between patient-matched samples maintained by optimal, flash-fro-

zen, and suboptimal, versus formalin-fixed paraffin-embedded samples $(F F P E)$, preservation methods. c Concordance of NS assay as a binary classifier from FFPE and frozen material
Fig. 5 Genomic and clinical correlates of EGFRvIII expression. a Significant EGFRvIII expression is exclusively found in tumors with amplification of EGFR. NS counts of EGFRvIII expression are plotted with respect to kinase domain counts. Blue circles denote samples with EGFR point mutation. Red denotes tumors with high-level amplification of the EGFR locus (aCGH $\log 2$ ratio $>2$ ). For two samples with high EGFRvIII expression, but $\log 2$ ratios below 2 (red arrows), aCGH demonstrates focal CNA in a pattern consistent with high-level gene amplification in a subpopulation of cells (and demonstrated by FISH for one of the two cases [43]). b Association between EGFR status and transcriptomal subclass. c Overall survival of patients stratified by EGFRvIII status. d Overall survival of patients stratified by EGFRvIII status excluding G-CIMP tumors, which are known to have a more favorable prognosis

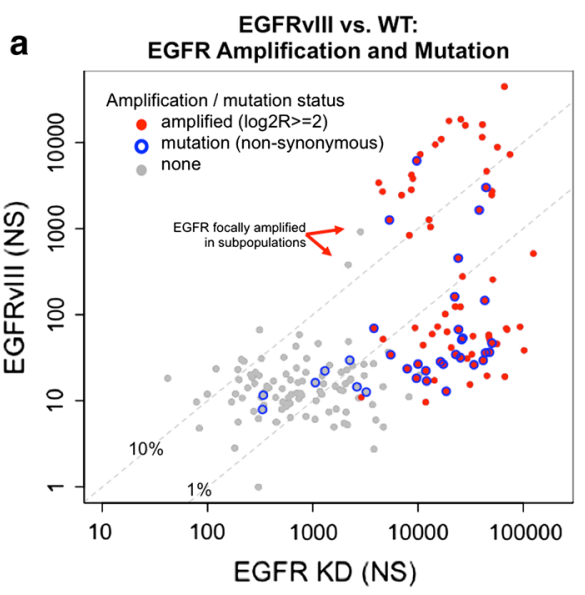

b

\begin{tabular}{|lccc|}
\hline & $\begin{array}{c}\text { EGFR } \\
\text { non-amp }\end{array}$ & $\begin{array}{c}\text { EGFR } \\
\text { amp }\end{array}$ & $\begin{array}{c}\text { EGFR } \\
\text { vIll+ }\end{array}$ \\
\hline Classical & 3 & 17 & 25 \\
Mesenchymal & 38 & 9 & 11 \\
Neural & 9 & 6 & 9 \\
Proneural & 52 & 4 & 2 \\
\hline
\end{tabular}

C

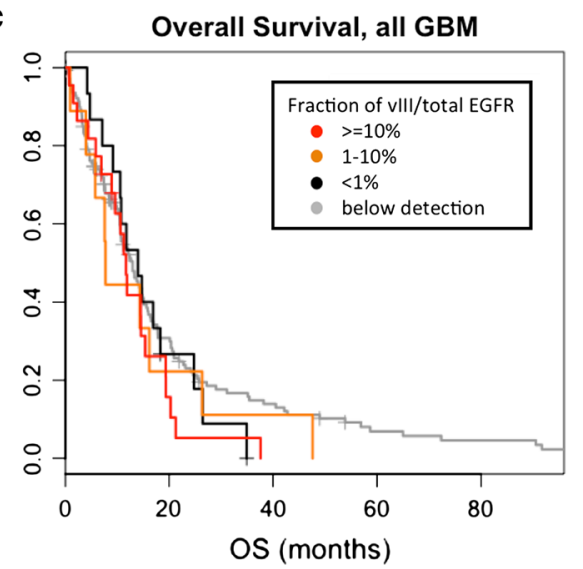

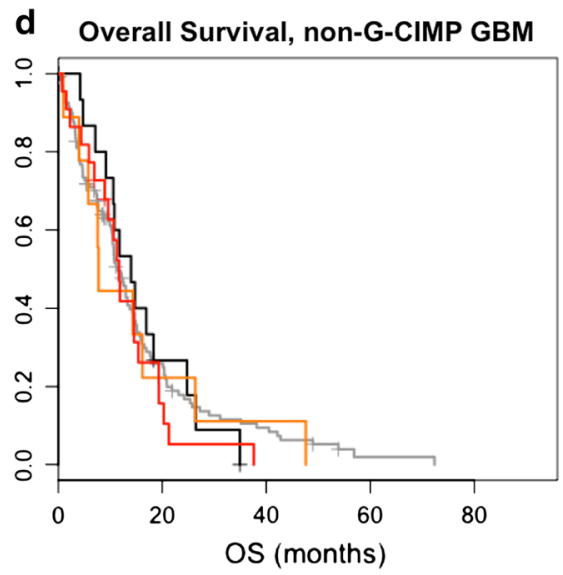

of 1,789 times (95\% CI for EGFRvIII 0-0.21\%). These results establish that GBMs rarely if ever express high levels of EGFRvIII in the absence of focal amplification of the locus. Additionally, there is no evidence of promiscuous low-level expression that one might expect if EGFRvIII were the result of common splicing variation.
EGFRvIII does not independently correlate with specific molecular and/or clinical features within EGFR-amplified GBM

EGFR amplification and EGFRvIII expression were both associated with the classical transcriptional subclass 


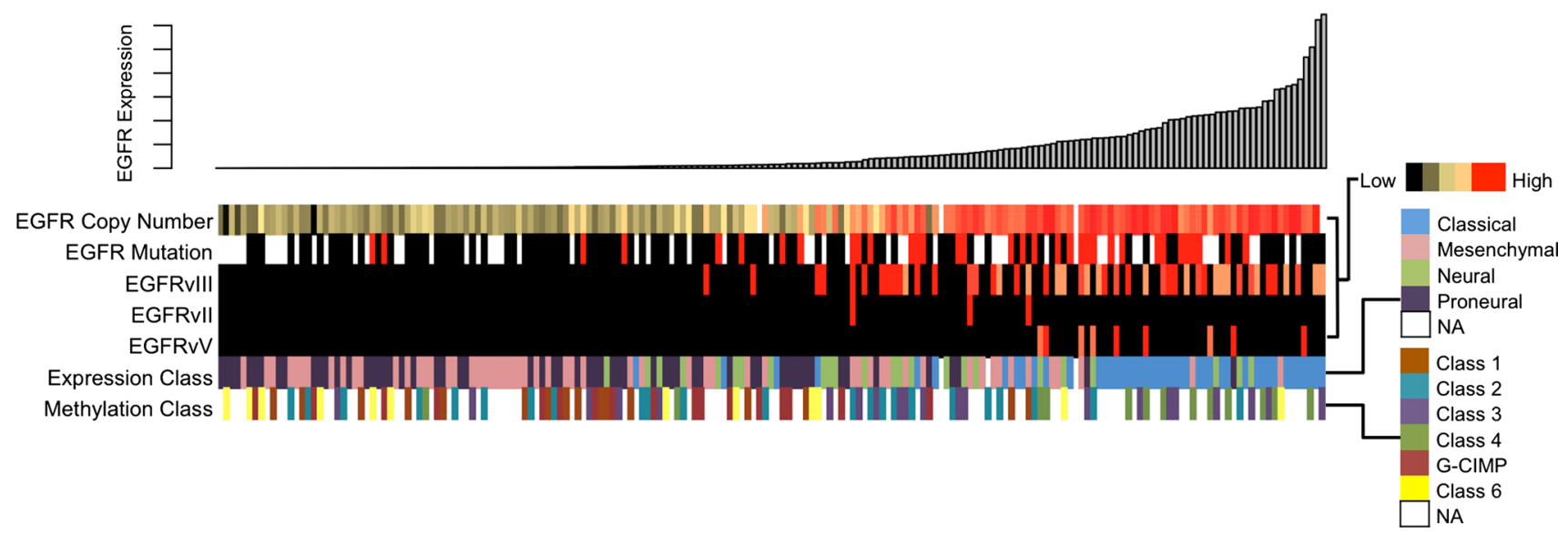

Fig. 6 Molecular context of EGFR alterations in GBM. From top to bottom EGFR mRNA expression, DNA copy number, deletion mutation expression, transcriptomal and methylation subclass are reported for each sample

(Fig. 5b). However, this association was not independently significant for EGFRvIII. Moreover, EGFRvIII positivity at any level was not predictive for overall survival in GBM (Fig. 5c). An apparent overall difference of long-term survivors disappears after excluding patients with the distinct phenotype of GBM CpG island hypermethylation (G-CIMP [34]) (Fig. 5d). Cox proportional hazards regression models fit either EGFRvIII counts, EGFR-WT counts, or EGFRvIII/EGFR ratio revealed no significant prognostic value for any of these parameters.

In a further effort to identify molecular features distinguishing EGFRvIII-mutant tumors from their wildtype EGFR-amplified counterparts, we utilized copy number, gene expression, and histopathological data for our TCGA sample set [5]. We first prospectively tested a limited set of selected molecular and histopathological parameters including small cell histology or pseudopalisading necrosis; deletion/mutation of TP53, NF1, PTEN, $C D K N 2 A, C D K N 2 C$, and $R B 1$; amplification of CDK4/6; mRNA expression of IL6 or LIF, MMP13 and BCL-XL. This demonstrated no statistically significant differences between EGFRvIII ${ }^{\mathrm{HI}}(n=20)$ and EGFRvIII-negative tumors $(n=37)$ within the EGFR-amplified subset (Supplemental Table S2). We then tested all TCGAmeasured variables using empirical Bayesian analysis and found no specific copy number events or mRNAs, miRNAs, or proteins whose differential expression between EGFRvIII-positive, and wild-type EGFRamplified tumors reached statistical significance. Similarly, no scored histopathological features were found to delineate mutant and wild-type samples by Chi-squared analysis.
Molecular and clinical features of GBMs harboring other RTK intragenic deletions

We screened other available molecular data to identify features that might be correlated with expression of EGFRvII, EGFRvV, and PDGFRA $\Delta 8,9$. As expected, PDGFRA $\Delta 8,9$ was seen at high levels only in the context of high-level PDGFRA amplification and its presence exclusively within the proneural expression subclass (Fig. 6; Supplemental Fig. S5c). Similarly, both EGFRvII and EGFRvV-positive tumors were invariably amplified for EGFR (Fig. 6). Somewhat surprisingly, we found that the three EGFRvIIexpressing tumors were all assigned to the mesenchymal expression subgroup (Supplemental Fig. S5a). By contrast, EGFR vV-positive tumors, particularly those exhibiting strong positivity, were primarily designated as classical, although mesenchymal and neural classifications were also seen, primarily for lower expressers (Supplemental Fig. S5b).

Detailed analysis of gene expression data revealed that, unlike for EGFRvIII, EGFRvII positivity correlated with a defined gene signature relative to wild-type EGFR-amplified tumors. In total, we observed 27 genes with statistically significant upregulation in EGFRvII tumors (Supplemental Table S3). Of these, 26/27 genes were similarly upregulated in mesenchymal tumors versus all others with two genes also upregulated in the setting of EGFR amplification, likely reflecting the transcriptional and genomic features of the three EGFRvII-positive tumors. Gene ontology (GO) analysis revealed significant correlations between the EGFRvII gene signature and chemokine activity, signal transduction, cellular locomotion (Supplemental Table S4). We did not identify genes or expression signatures similarly 
associated with either EGFR $v V$ or PDGFRA $\Delta 8,9$ positivity relative to wild-type receptor-amplified tumors, nor were specific miRNAs or copy number events correlated with any of the three deletion mutants. Moreover, consistent with earlier work demonstrating the frequent co-occurrence of EGFR vII and vV with vIII [10], we found that all EGFR vII-positive tumors and $44 \%$ (4/9) of EGFR vVpositive tumors in our sample set also expressed high levels of EGFR vIII (Fig. 6). Finally, overall survival was not significantly different between either EGFR vII or vV-mutant tumors and all others (Supplemental Fig. S6a-S6b). And while Kaplan-Meier analysis identified poorer outcomes for PDGFRA $\Delta 8,9$ tumors $(p=0.0257)$, sample size limits the robustness of this finding (Supplemental Fig. S6c).

\section{Discussion}

The Cancer Genome Atlas GBM initiative has recently completed analysis of a molecularly and clinically annotated dataset of unprecedented detail for over 500 tumors [5]. This project was initiated in 2006, before the advent of high-throughput DNA and RNA sequencing technologies. As a result, the initial TCGA marker paper in 2008 had no direct measure of intragenic deletion mutations despite these being the most common forms of RTK activation in GBM [44]. Our study aims to provide this annotation for 189 TCGA tumors, quantified by Nanostring and verified for EGFRvIII quantitatively by RT-PCR. As technology has advanced, TCGA has subsequently performed RNA sequencing for 164 of the most recent cases, 47 overlapping our NS dataset. Together, the NS and RNA-seq data provide a quantitative annotation of common RTK deletion variants for 306 tumors. The ability to cross-reference expression levels of EGFRvIII and other RTK deletions against the clinical and detailed molecular data in TCGA provides a valuable resource to better understand the molecular context in which these mutations are found.

We found no prognostic significance of EGFRvIII expression in the primary GBMs comprising TCGAs dataset. This is consistent with some prior studies performed on independent datasets [2, 15,24]. Our global analysis of molecular correlates of EGFRvIII and other deletion mutations revealed that, for the most part, tumors with these mutations were also not distinguished by specific molecular features compared to their wild-type RTK-amplified counterparts. This analysis does not imply that EGFRvIII expression has no molecular effects, but rather that detecting these effects in the TCGA data will require prospective testing of select hypotheses. The TCGA dataset also does not reflect differences in subcellular localization, posttranslational modification, or degradation of EGFR protein, any or all of which might be impacted distinctly by
vIII mutation [7, 14, 25]. Nonetheless, the global similarity of EGFR-amplified tumors, whether EGFRvIII positive or negative, suggests that common features are shared by GBMs with EGFR activation by any means, and that neomorphic functions specific to EGFRvIII may not be strongly influential on the tumor phenotypes measured here. In contrast, EGFRvII-expressing GBMs do appear to have an expression signature distinct from most other EGFR-amplified tumors. It is likely that this finding reflects the association of vII mutation with mesenchymal rather than classical transcriptional subclass, as 26/27 EGFRvII signature genes $(96 \%)$ were also associated with non-vIIexpressing mesenchymal GBMs in the same analysis.

Because RTK mutations are typically associated with gene amplification in GBM, there can be a wide range of expression of mutant and wild-type alleles [10], and these levels may vary tumor-to-tumor and even cell-to-cell [19, 33]. Earlier work has shown that multiple mutations can affect a single EGFR allele [10]. Recent analysis of TCGA RNA-seq data revealed that multiple EGFR deletion and point mutations were often expressed in the same tumor at different allelic frequencies [5]. We observed a high rate of co-occurrence between different EGFR deletion mutants in our sample set- $100 \%$ of EGFRvII and $44 \%$ of EGFRvVpositive tumors also harbored EGFRvIII. The biological significance of multiple coincident EGFR deletion mutations in the same tumor remains unclear. Interestingly, some evidence supports the possibility of functional heterodimerization involving mutant and wild-type receptors, which may play a driving role in the maintenance of EGFRvIII as a minority species in a transformed cell [11,25].

In addition to providing a molecular annotation resource, this report describes a transcript-based quantitative assessment of EGFRvIII, along with other deletion mutants operative from a relatively small amount of biomaterial. Our Nanostring-based assay exhibited notable linearity even at low levels of transcript expression and performed well in the context of FFPE starting material. This latter finding, consistent with a number of prior studies, likely reflects the absence of PCR in the Nanostring workflow. Indeed, such signal amplification can accentuate systematic error in quantitative measurements, particularly in the context of compromised starting material. Methods for the routine detection of RTK deletion mutants like EGFRvIII from surgical biopsy material remain poorly standardized and nonquantitative. Immunohistochemistry and/or RT-PCR are the predominant assays used in the clinical setting, with results typically interpreted in a binary fashion as either "positive" or "negative". While such readouts are practical for certain applications and are currently less expensive, they do not accurately capture the molecular and cellular heterogeneity known to characterize GBM, nor are they readily quantifiable. Moreover, recent analysis has shown that multiple 
EGFR point and deletion mutations can be expressed in the same tumor at different allelic frequencies [5].

Overall, our findings agree with prior literature, both in the proportion of cases where Nanostring was suggestive of EGFRvIII-24\% of total and $54 \%$ of EGFRamplified-as well as the proportion of high-level expressers $(10.6 \%$ overall) $[3,51]$. These figures include cases in which EGFRvIII was detected in $<1 \%$ of EGFR transcripts, where the biological significance and contribution of technical noise is unknown. Available RNA-seq data from overlapping TCGA samples provided strong cross-validation, as detectable reads for EGFRvIII were present in all but one of the samples designated $>1 \%$ by Nanostring. Similar correlations were observed for EGFRvII, EGFRvV, and PDGFRA $\Delta 8,9$, albeit on fewer samples. The higher sensitivity of the Nanostring assay to detect mutant transcripts at low expression levels may be related to better coverage depth. In all cases, Nanostring provided markedly higher read counts than RNA-seq (typically 50- to 100 -fold greater). Next-generation sequencing costs can only be expected to fall in the coming years, enabling higher read counts routinely. Nevertheless, the limited tissue specimens available in the clinical setting may be insufficient to supply the microgram quantities of RNA typically required for transcriptome sequencing, and a significant proportion of clinical material is FFPE. Thus, assay platforms that are both cost- and resource-effective will continue play central roles in clinical management. Additionally, the ability of the Nanostring nCounter to assess up to 800 mRNAs simultaneously, while not comprehensive, should allow the multiplexing of RTK deletion mutants with a number of other transcripts and gene expression signatures of interest without increasing the required biomaterial.

RTK deletion mutants, along with their wild-type receptors, remain therapeutic targets of considerable potential for GBM. The lack of encouraging clinical results with RTK inhibition thus far may reflect, in part, inadequate drug penetration, lack of molecular stratification in clinical trials and signaling feedback mechanisms [8, 18, 27]. Cellular and molecular heterogeneity involving wild-type and mutant RTK composition, as we observed in this study, likely complicates strategies to effectively inhibit oncogenic signaling. Indeed, investigations carried out in vitro and in human patients indicate that the inhibitor sensitivity profiles of wild-type EGFR and EGFRvIII are distinct [46]. In this respect, our findings and those of others support the notion that a successful therapeutic strategy will require the effective inhibition of both mutant and wild-type receptor at concentrations achievable in the target tissue. Indeed, incomplete targeting of EGFR isoforms could simply drive tumor evolution toward a cellular population expressing an untargeted (resistant) variant. For loci that are commonly amplified in GBM, "quantitative genotyping" of the amplicons and their contained mutations may be a requirement to unambiguously establish their value as predictive and prognostic markers, particularly if established pathogenic mutations exist as minority species. Consequently, methodologies such as those described in this report may prove vitally important to standard clinical practice.

Acknowledgments This work was supported by TCGA Pilot Phase Cancer Genome Characterization Center at MSKCC, NIH/NCI U24 CA126543. The results published here are in part based upon data generated by The Cancer Genome Atlas pilot project established by the NCI and NHGRI. Information about TCGA and the investigators and institutions constituting the TCGA research network can be found at http://cancergenome.nih.gov.

Open Access This article is distributed under the terms of the Creative Commons Attribution License which permits any use, distribution, and reproduction in any medium, provided the original author(s) and the source are credited.

\section{References}

1. Al-Nedawi K, Meehan B, Micallef J et al (2008) Intercellular transfer of the oncogenic receptor EGFRvIII by microvesicles derived from tumour cells. Nat Cell Biol 10:619-624. doi:10.1038/ncb1725

2. Aldape KD, Ballman K, Furth A et al (2004) Immunohistochemical detection of EGFRvIII in high malignancy grade astrocytomas and evaluation of prognostic significance. J Neuropathol Exp Neurol 63:700-707

3. Biernat W, Huang H, Yokoo H, Kleihues P, Ohgaki H (2004) Predominant expression of mutant EGFR (EGFRvIII) is rare in primary glioblastomas. Brain Pathol 14:131-136

4. Brennan C, Momota H, Hambardzumyan D et al (2009) Glioblastoma subclasses can be defined by activity among signal transduction pathways and associated genomic alterations. PLoS One 4:e7752. doi:10.1371/journal.pone.0007752

5. Brennan CW, Verhaak RG, McKenna A et al (2013) The somatic genomic landscape of glioblastoma. Cell 155:462-477. doi:10.1016/j.cell.2013.09.034

6. Chen WS, Lazar CS, Lund KA et al (1989) Functional independence of the epidermal growth factor receptor from a domain required for ligand-induced internalization and calcium regulation. Cell 59:33-43

7. Cho J, Pastorino S, Zeng Q et al (2011) Glioblastoma-derived epidermal growth factor receptor carboxyl-terminal deletion mutants are transforming and are sensitive to EGFR-directed therapies. Cancer Res 71:7587-7596. doi:10.1158/0008-5472.CAN-11-0821

8. Dunn GP, Rinne ML, Wykosky J et al (2012) Emerging insights into the molecular and cellular basis of glioblastoma. Genes Dev 26:756-784. doi:10.1101/gad.187922.112

9. Ekstrand AJ, Sugawa N, James CD, Collins VP (1992) Amplified and rearranged epidermal growth factor receptor genes in human glioblastomas reveal deletions of sequences encoding portions of the $\mathrm{N}$ - and/or C-terminal tails. Proc Natl Acad Sci USA 89:4309-4313

10. Frederick L, Wang XY, Eley G, James CD (2000) Diversity and frequency of epidermal growth factor receptor mutations in human glioblastomas. Cancer Res 60:1383-1387

11. Gajadhar AS, Bogdanovic E, Munoz DM, Guha A (2012) In Situ analysis of mutant EGFRs prevalent in glioblastoma multiforme 
reveals aberrant dimerization, activation, and differential response to anti-EGFR targeted therapy. Mol Cancer Res 10:428440. doi:10.1158/1541-7786.MCR-11-0531

12. Gan HK, Kaye AH, Luwor RB (2009) The EGFRvIII variant in glioblastoma multiforme. J Clin Neurosci 16:748-754. doi:10.1016/j.jocn.2008.12.005

13. Geiss GK, Bumgarner RE, Birditt B et al (2008) Direct multiplexed measurement of gene expression with color-coded probe pairs. Nat Biotechnol 26:317-325. doi:10.1038/nbt1385

14. Grandal MV, Zandi R, Pedersen MW, Willumsen BM, van Deurs B, Poulsen HS (2007) EGFRvIII escapes down-regulation due to impaired internalization and sorting to lysosomes. Carcinogenesis 28:1408-1417. doi:10.1093/carcin/bgm058

15. Heimberger AB, Hlatky R, Suki D et al (2005) Prognostic effect of epidermal growth factor receptor and EGFRvIII in glioblastoma multiforme patients. Clin Cancer Res 11:1462-1466. doi:10.1158/1078-0432.CCR-04-1737

16. Horvath S, Zhang B, Carlson M et al (2006) Analysis of oncogenic signaling networks in glioblastoma identifies ASPM as a molecular target. Proc Natl Acad Sci USA 103:17402-17407. doi :10.1073/pnas.0608396103

17. Huang PH, Mukasa A, Bonavia R et al (2007) Quantitative analysis of EGFRvIII cellular signaling networks reveals a combinatorial therapeutic strategy for glioblastoma. Proc Natl Acad Sci USA 104:12867-12872. doi:10.1073/pnas.0705158104

18. Huse JT, Holland E, Deangelis LM (2013) Glioblastoma: molecular analysis and clinical implications. Annu Rev Med 64:59-70. doi:10.1146/annurev-med-100711-143028

19. Inda MM, Bonavia R, Mukasa A et al (2010) Tumor heterogeneity is an active process maintained by a mutant EGFR-induced cytokine circuit in glioblastoma. Genes Dev 24:1731-1745. doi:10.1101/gad.1890510

20. Kuan CT, Wikstrand CJ, Bigner DD (2001) EGF mutant receptor vIII as a molecular target in cancer therapy. Endocr Relat Cancer 8:83-96

21. Kumabe T, Sohma Y, Kayama T, Yoshimoto T, Yamamoto T (1992) Amplification of alpha-platelet-derived growth factor receptor gene lacking an exon coding for a portion of the extracellular region in a primary brain tumor of glial origin. Oncogene 7:627-633

22. Lal A, Glazer CA, Martinson HM et al (2002) Mutant epidermal growth factor receptor up-regulates molecular effectors of tumor invasion. Cancer Res 62:3335-3339

23. Lee JC, Vivanco I, Beroukhim R et al (2006) Epidermal growth factor receptor activation in glioblastoma through novel missense mutations in the extracellular domain. PLoS Med 3:e485. doi:10.1371/journal.pmed.0030485

24. Liu L, Backlund LM, Nilsson BR et al (2005) Clinical significance of EGFR amplification and the aberrant EGFRvIII transcript in conventionally treated astrocytic gliomas. J Mol Med 83:917-926. doi:10.1007/s00109-005-0700-2

25. Luwor RB, Zhu HJ, Walker F et al (2004) The tumor-specific de2-7 epidermal growth factor receptor (EGFR) promotes cells survival and heterodimerizes with the wild-type EGFR. Oncogene 23:6095-6104. doi:10.1038/sj.onc.1207870

26. Mellinghoff IK, Wang MY, Vivanco I et al (2005) Molecular determinants of the response of glioblastomas to EGFR kinase inhibitors. N Engl J Med 353:2012-2024. doi:10.1056/NEJ Moa051918

27. Mellinghoff IK, Schultz N, Mischel PS, Cloughesy TF (2012) Will kinase inhibitors make it as glioblastoma drugs? Curr Top Microbiol Immunol 355:135-169. doi:10.1007/82_2011_178

28. Mitsudomi T, Yatabe Y (2010) Epidermal growth factor receptor in relation to tumor development: EGFR gene and cancer. FEBS J 277:301-308. doi:10.1111/j.1742-4658.2009.07448.x

29. Mizoguchi M, Betensky RA, Batchelor TT, Bernay DC, Louis DN, Nutt CL (2006) Activation of STAT3, MAPK, and AKT in malignant astrocytic gliomas: correlation with EGFR status, tumor grade, and survival. J Neuropathol Exp Neurol 65:11811188. doi:10.1097/01.jnen.0000248549.14962.b2

30. Nagane M, Coufal F, Lin H, Bogler O, Cavenee WK, Huang HJ (1996) A common mutant epidermal growth factor receptor confers enhanced tumorigenicity on human glioblastoma cells by increasing proliferation and reducing apoptosis. Cancer Res 56:5079-5086

31. Network $T$ (2008) Comprehensive genomic characterization defines human glioblastoma genes and core pathways. Nature 455:1061-1068. doi:10.1038/nature07385

32. Nicholas MK, Lukas RV, Jafri NF, Faoro L, Salgia R (2006) Epidermal growth factor receptor-mediated signal transduction in the development and therapy of gliomas. Clin Cancer Res 12:7261-7270. doi:10.1158/1078-0432.CCR-06-0874

33. Nishikawa R, Sugiyama T, Narita Y, Furnari F, Cavenee WK, Matsutani M (2004) Immunohistochemical analysis of the mutant epidermal growth factor, deltaEGFR, in glioblastoma. Brain Tumor Pathol 21:53-56

34. Noushmehr H, Weisenberger DJ, Diefes K et al (2010) Identification of a $\mathrm{CpG}$ island methylator phenotype that defines a distinct subgroup of glioma. Cancer Cell 17:510-522. doi:10.1016/j.ccr.2010.03.017

35. Okada H, Kohanbash G, Zhu X et al (2009) Immunotherapeutic approaches for glioma. Crit Rev Immunol 29:1-42

36. Ozawa T, Brennan CW, Wang L et al (2010) PDGFRA gene rearrangements are frequent genetic events in PDGFRA-amplified glioblastomas. Genes Dev 24:2205-2218. doi:10.1101/gad.1972310

37. Phillips HS, Kharbanda S, Chen R et al (2006) Molecular subclasses of high-grade glioma predict prognosis, delineate a pattern of disease progression, and resemble stages in neurogenesis. Cancer Cell 9:157-173. doi:10.1016/j.ccr.2006.02.019

38. Pines G, Huang PH, Zwang Y, White FM, Yarden Y (2010) EGFRvIV: a previously uncharacterized oncogenic mutant reveals a kinase auto-inhibitory mechanism. Oncogene 29:58505860. doi:10.1038/onc.2010.313

39. Prigent SA, Nagane M, Lin H et al (1996) Enhanced tumorigenic behavior of glioblastoma cells expressing a truncated epidermal growth factor receptor is mediated through the Ras-Shc-Grb2 pathway. J Biol Chem 271:25639-25645

40. Rand V, Huang J, Stockwell T et al (2005) Sequence survey of receptor tyrosine kinases reveals mutations in glioblastomas. Proc Natl Acad Sci USA 102:14344-14349. doi:10.1073/p nas.0507200102

41. Smyth GK (2004) Linear models and empirical bayes methods for assessing differential expression in microarray experiments. Stat Appl Genet Mol Biol 3: Article3 Doi: 10.2202/1544-6115.1027

42. Sugawa N, Ekstrand AJ, James CD, Collins VP (1990) Identical splicing of aberrant epidermal growth factor receptor transcripts from amplified rearranged genes in human glioblastomas. Proc Natl Acad Sci USA 87:8602-8606

43. Szerlip NJ, Pedraza A, Chakravarty D et al (2012) Intra-tumoral heterogeneity of receptor tyrosine kinases EGFR and PDGFRA amplification in glioblastoma defines subpopulations with distinct growth factor response. Proc Natl Acad Sci USA 109:3041-3046. doi:10.1073/pnas.1114033109

44. TCGA (2008) Comprehensive genomic characterization defines human glioblastoma genes and core pathways. Nature 455:10611068. doi: $10.1038 /$ nature 07385

45. Verhaak RG, Hoadley KA, Purdom E et al (2010) Integrated genomic analysis identifies clinically relevant subtypes of glioblastoma characterized by abnormalities in PDGFRA, IDH1, EGFR, and NF1. Cancer Cell 17:98-110. doi:10.1016/j.ccr.2009.12.020

46. Vivanco I, Robins HI, Rohle D et al (2012) Differential sensitivity of glioma- versus lung cancer-specific EGFR mutations to EGFR 
kinase inhibitors. Cancer Discov 2:458-471. doi:10.1158/21598290.CD-11-0284

47. Voldborg BR, Damstrup L, Spang-Thomsen M, Poulsen HS (1997) Epidermal growth factor receptor (EGFR) and EGFR mutations, function and possible role in clinical trials. Ann Oncol 8:1197-1206

48. Wells A, Welsh JB, Lazar CS, Wiley HS, Gill GN, Rosenfeld MG (1990) Ligand-induced transformation by a non-internalizing epidermal growth factor receptor. Science 247:962-964

49. Wiedemeyer R, Brennan C, Heffernan TP et al (2008) Feedback circuit among INK4 tumor suppressors constrains human glioblastoma development. Cancer Cell 13:355-364. doi:10.1016/j.ccr.2008.02.010

50. Wu JL, Abe T, Inoue R, Fujiki M, Kobayashi H (2004) IkappaBalphaM suppresses angiogenesis and tumorigenesis promoted by a constitutively active mutant EGFR in human glioma cells. Neurol Res 26:785-791. doi:10.1179/016164104225014139

51. Yoshimoto K, Dang J, Zhu S et al (2008) Development of a real-time RT-PCR assay for detecting EGFRvIII in glioblastoma samples. Clin Cancer Res 14:488-493. doi:10.1158/1078-0432.CCR-07-1966 